\title{
La formule des Hollandais ou le conformisme dans l'enseignement
}

\section{H. GONIN}

42, villa Brimborion 92190 Meudon
On analyse, dans cet article, la façon dont les ondes de compression nées du choc d'un marteau sur une tige sont utilisées pour la pénétration au cours d'une succession de réflexions aux extrémités. Le nombre d'ondes réfléchies utilisables pour la pénétration dépend du rapport entre la résistance à la pénétration en pointe dans le millieu rigide-plastique considéré, $q_{d}$, et la valeur maximale de la contrainte incidente $\sigma_{\text {ima }}$

On se livre, par la même occasion, à une critique argumentée de la formule de battage dite « des Hollandais $m$, basée sur un raisonnement qui ne respecte pas la conservation de l'énergie et qui est donc en contradiction avec les principes fondamentaux de la Mécanique rationnelle.

\section{Dutch Formula or teaching mistakes}

In this article, the tip-penetration due to incident and reflected stress-waves issued from the impact of one hammer on a rod is analysed. The number of reflected stress-waves used for penetration is conditioned by the value of the quotient of tipresistance $q_{d}$ characterizing the rigid-plastic medium considered by maximum value of the incident stress-wave $\sigma_{\text {it }}$.

The opportunity is taken for a rational review of the driving formula called $\alpha$ Dutch Formula $»$, based on a demonstration leaving out conservation of energy, that is therefore contradictory with basic principles of the rational Mechanics. 
L'indépendance d'esprit est un don précieux, qui conduit à n'accepter que ce qui a été assimilé au prix d'un effort personnel de réflexion et de « re-découverte m, évitant ainsí le piège confortable des préjugés. Le cas de la fameuse a formule des Hollandais $x$, qui concerne l'enfoncement par battage, et dont la “demonstration 1 est exposée sans discernement dans de nombreux traités techniques sur le sujet, constitue une bonne illustration des méfaits du conformisme dans l'enseignement. Pour bien le comprendre, il nous faut revenir au principe de base de la conservation de la quantité de mouvement, appliqué aux chocs entre corps déformables. Ce qui suit paraîtra élémentaire aux théoriciens de la mécanique, mais je m’adresse, sans prétention, à des ingénieurs ou techniciens de génie civil de formation très générale, dont je fais évidemment partie.

\section{La quantité de mouvement}

Newton, ce grand génie, a su, le premier, définir la masse d'un corps (m) et sa quantité de mouvement (mv). La notion de force intervient pour caractériser dans le temps les variations de la quantité de mouvement

$$
\Delta(m v)=F \times \Delta t
$$

Ce retour à la source met en évidence la lumineuse simplicité des concepts de base, fruits de l'observation, et nous nous émerveillons encore, trois siècles plus tard, devant leur extraordinaire fécondité, en dépit de leur récente remise en cause pour «se mettre en accord avec l'ensemble des faits observés n, suivant l'expression même d'Einstein.

De (1) découle, en faisant intervenir la notion de vecteur, si m est invariable, la relation classique:

$$
\vec{F}=m \cdot \frac{\overrightarrow{d v}}{d t}=m, \vec{\gamma}
$$

Puis le travail élémentaire dW étant défini comme le produit scalaire d'une Force $\vec{F}$ par son déplacement infinitésimal ds:

$$
\frac{d w}{d t}=\vec{F} \cdot \frac{\vec{d} s}{d t}=m \cdot \frac{\vec{d} v}{d t} \cdot \vec{v}=\frac{d}{d t}\left(1 / 2 m v^{2}\right)
$$

Le travail utilisé pour amener une masse $m$ de l'immobilité à la vitesse $\vec{v}$ est mesuré par son énergie cinétique $1 / 2 \mathrm{mv}^{2}$.

Si la force $\vec{F}$ dérive d'une énergie potentielle

$\vec{F}=-$ grad $\Psi$, il est immédiat que la quantité $1 / 2 \mathrm{mv}^{2}$ $+\Psi$ est constante pendant le mouvement : la somme de l'ènergie cinétique et de l'énergie potentielle est constante (conservation de l'énergie).

Ce qui précède s'accorde bien avec le concept de corps formés d'un assemblage indéformable de points massiques: par exemple, le champ de la pesanteur exerce sur un corps de masse m supposé indéformable une force $\vec{F}=m \vec{g}$ appliquée à son centre de gravité, et pour un corps en chute libre d'une hauteur $\mathrm{H}, 1 / 2 \mathrm{mv}^{2}=$ $\mathrm{mgH}$ : énergie cinétique gagnée ef énergie potentielle perdue sont égales. Mais cela devient moins clair lorsqu' une force s'exerce localement sur un corps déformable : c'est le cas de deux corps, animés de vitesses différentes, dont les trajectoires se rencontrent et qui s'entrechoquent.
Un corps soumis à une action extérieure subit des déformations, donc il échange de l'énergie avec l'extérieur. Pour un corps élancé supposé élastique, en petites déformations, le principe de linéarité externe est respecté, et le théorème de Clayperon nous apprend que l'énergie de déformation, pour passer d'un état initial non sollicité à un état d'équilibre sous un changement $F$, est égal à la moitié du travail des forces extérieures produisant la déformation totale (voir l'exemple de la figure 1).

Que se passe-t-il donc pour le choc de deux corps élastiques, où le chargement $F$ apparait au moment de leur rencontre, sans passer par une succession d'états d'équilibre? On peut penser que la moitié de l'énergie étant consacrée à déformer les corps entrés en contact. l'autre moitié l'est à modifier leurs quantités de mouvement, le bilan total restant constant. On distinguera donc, pour un corps ayant subi un choc, la vitesse des points massiques du corps non déformé et la "vitesse particulaire x obtenue en un point par la déformation consécutive au choc. Mais les principes de Newton restent applicables; si aucune force exterieure n'intervient, il y a conservation à la fois de la quantité de mouvement et de l'énergie du système : le « chargement » F résultant du choc est le même, dans son intensité et dans sa durée, pour chacun des deux corps qui se sont entrechoqués: ce que l'un perd, l'autre le gagne!

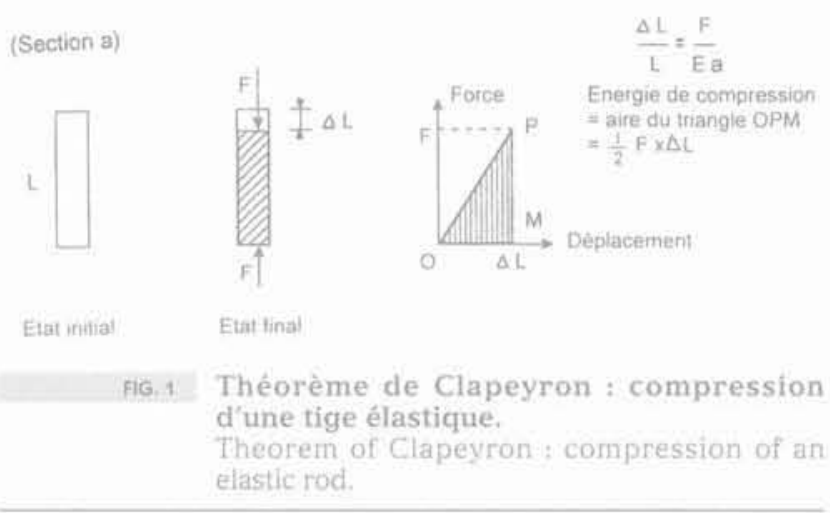
2

\section{La formule des Hollandais}

Les Hollandais sont, depuis des siècles, des batteurs de pieux émérites, et c'est sans doute pour cela que cette fameuse formule, destinée à vérifier la force portante de pieux battus, leur est attribuée. Si elle n'est plus utilisée pour cela, même sous des formes corrigées (formules de Hiley et autres), elle l'est encore beaucoup pour apprécier la u résistance dynamique » des sols à l'aide de pénétromètres dynamiques, jusqu'à apparaitre dans nos normes !

La formule des Hollandais résulte d'un raisonnement ; elle n'est ni déduite de constatations expérimentales, ni confirmée par les observations, quoi qu'en disent ses utilisateurs inconditionnels. Ce raisonnement est le suivant :

Une masse $M$ (mouton) tombe sur une tige de masse totale $\mathrm{m}$ d'une hauteur $\mathrm{H}$ (voir figure 2). La vitesse à l'impact est $V_{M}=\sqrt{2 g H}$.

Immédiatement avant le choc, la quantité de mouvement totale est égale à $M V_{M}$, la tige étant immobile. 


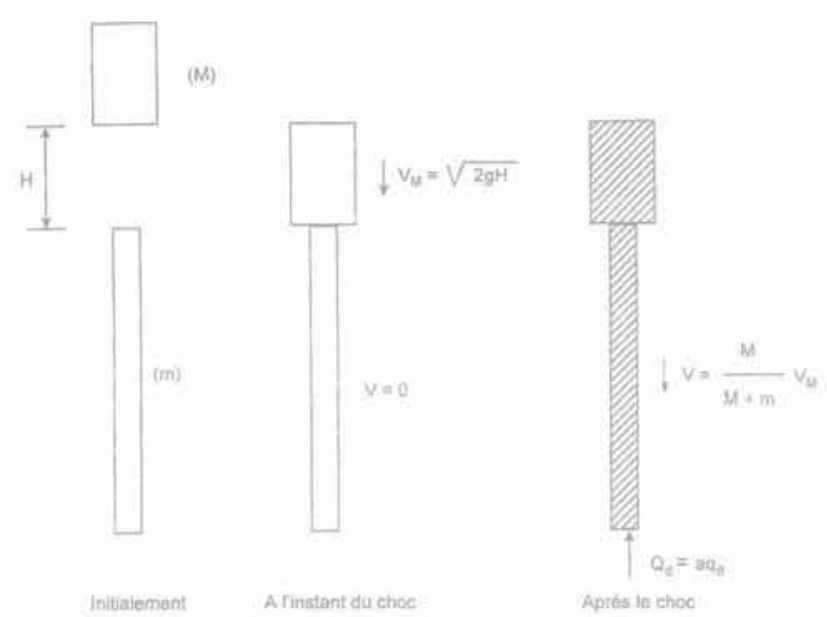

FIG. 2 Formule des Hollandais. Dutch Formula

Après le choc, la conservation de la quantité de mouvement (aucune action extérieure autre que la gravité, d'effet négligeable) permet de calculer la vitesse $V$ de l'ensemble du mouton et de la tige devenus solidaires :

$$
V=\frac{M}{M+m} V_{M}
$$

L'énergie cinétique de la masse $(\mathrm{M}+\mathrm{m})$ lancée à la vitesse $V$ est donc :

$$
\frac{1}{2}(M+m) V^{2}=\frac{1}{2} \frac{M^{2} V_{M}^{2}}{M+m}=\frac{1}{2} M V_{M !}^{2} \times \frac{M}{M+m}=M g H \times \frac{M}{M+m}
$$

C'est cette énergie qui est utilisée pour la pénétration. Si la résistance du milieu est constante et égale à $Q_{d}$ et l'enfoncement égal à $\mathrm{S}$, on peut écrire:

$$
Q_{d} \times S=M g H \times \frac{M}{M+m}
$$

D'où $Q_{\text {j }}$ en fonction de l'énergie et de la masse du mouton, de l'enfoncement et de la masse totale des tiges.

Ce raisonnement n'est ni approché, ni valable sous conditions: il est faux. car il ne respecte pas les principes fondamentaux de la Mécanique rationnelle. Quelle erreur que de croire qu'il puisse être validé par l'expérience!

\section{3}

\section{Choc d'un mouton sur une tige: analyse respectant les principes de Newton}

Le concept de corps indéformables n'est qu'une abstraction sans réalité ; pour étudier un choc, rencontre entre deux corps animés de vitesses différentes, on ne peut évidemment pas faire abstraction de leur déformabilité : c'est elle qui permet un échange d'énergie. L'hypothèse de corps parfaitement élastiques, homogènes et isotropes, permet l'abord théorique du problème. La réversibilité effort-déformation annule toute fonction dissipative et la totalité de l'énergie reçue ou fournie par un corps lors d'un choc l'est sous forme d'énergie cinétique, d'une part, et d'ẻnergie de déformation, d'autre part; d'après le théorème de Clapey- ron (voir plus haut), ces deux parts sont égales. Tant que dure le contact, l'énergie perdue par l'un des corps est absorbée par l'autre; dès que le contact cesse, l'échange est interrompu.

Pour mieux comprendre, considérons un cas simple: un mouton et une tige constitués de tiges cylindriques identiques, verticales et coaxiales, de section droite a, de matière homogène, isotrope et élastique, de module d'Young E, de masse volumique p et de longueurs respectives L et kL. La tige du haut tombe d'une hauteur $H$ sur la tige du bas. On néglige les déformations horizontales des deux tiges.

La vitesse du mouton à l'impact est $V_{M}=\sqrt{2 g H}$, la tige étant immobile; cette dernière repose à son extrémité basse sur un milieu de comportement rigide-plastique qui offre une résistance $Q_{d}=a q_{d}$ (voir figure 3).

A linstant du choc, il y a modification de la quantité de mouvement simultanément dans le mouton et dans la tige : il nait donc une force de compression $F$ à leur contact, supposé parfait sur toute la section droite. Par raison de symétrie, dans les premiers instants après le choc, la vitesse particulaire au contact est $\mathrm{V}_{\mathrm{M}} / 2$; si, au temps dt, la longueur cornprimée de la tige est cdt, la relation $(1)$ fondamentale $\Delta(\mathrm{mv})=\mathrm{F} \times \Delta \mathrm{t}$ nous permet d'écrire: $F \times d t=\rho a c d t \times \frac{V_{M}}{2}(2)$.

La déformation de la tige étant $\frac{V_{M}}{2} d t$, la loi de Hooke donne: $\frac{V_{M}}{2} d t=\frac{F}{a E} \times c d t(3)$.

de (2) et (3), on tire : $c=\left(\frac{E}{\rho}\right)^{1 / 2}$.

Le principe de Newton et la loi de Hooke ont donc pour conséquence directe que l'onde de compression née du choc, quỉ véhicule une discontinuité de vitesse constante égale à $\frac{V_{M}}{2}$, se propage en sens opposés dans le mouton et dans la tige avec une célérité constante c qui ne dépend que des propriétés de la matière qui les constitue. La longueur de la partie comprimée croit à une vitesse égale à $2 c$, son raccourcissement croit à la vitesse $V_{M}$; au bout d'un temps $t=\frac{L}{c}$, sa longueur est 2L, sa quantité de mouvement devient égale à celle du mouton à l'impact: $2 a L \rho \times \frac{V_{M}}{2}=a L \rho V_{M}$.

Cette flèche comprimée, lancée vers le bas dans la tige à la vitesse c, contient alors toute l'énergie du mouton, sous forme d'énergie de compression et d'énergie cinétique, qui sont égales :

- énergie de compression :

$$
\frac{1}{2} F \times V_{M} \times \frac{L}{c}=\frac{1}{4} a L \frac{E}{c^{2}} V_{M}^{2}=\frac{1}{4} a L \rho V_{M}^{2}
$$

- énergie cinétique : $\frac{1}{2} \times 2 a L \rho \times \frac{V_{M}^{2}}{4}=\frac{1}{4} a L \rho V_{M}^{2}$

- énergie totale :

$$
\frac{1}{2} a L \rho V_{M}^{2}=\frac{1}{2} M V_{M}^{2}
$$

Elle ne peut donc que poursuivre (vers le bas) son chemin dans la tige avec une célérité égale à c, laissant le mouton immobile et complètement vidé de son énergie à partir du temps $t=\frac{2 L}{C}$ après le choc. 


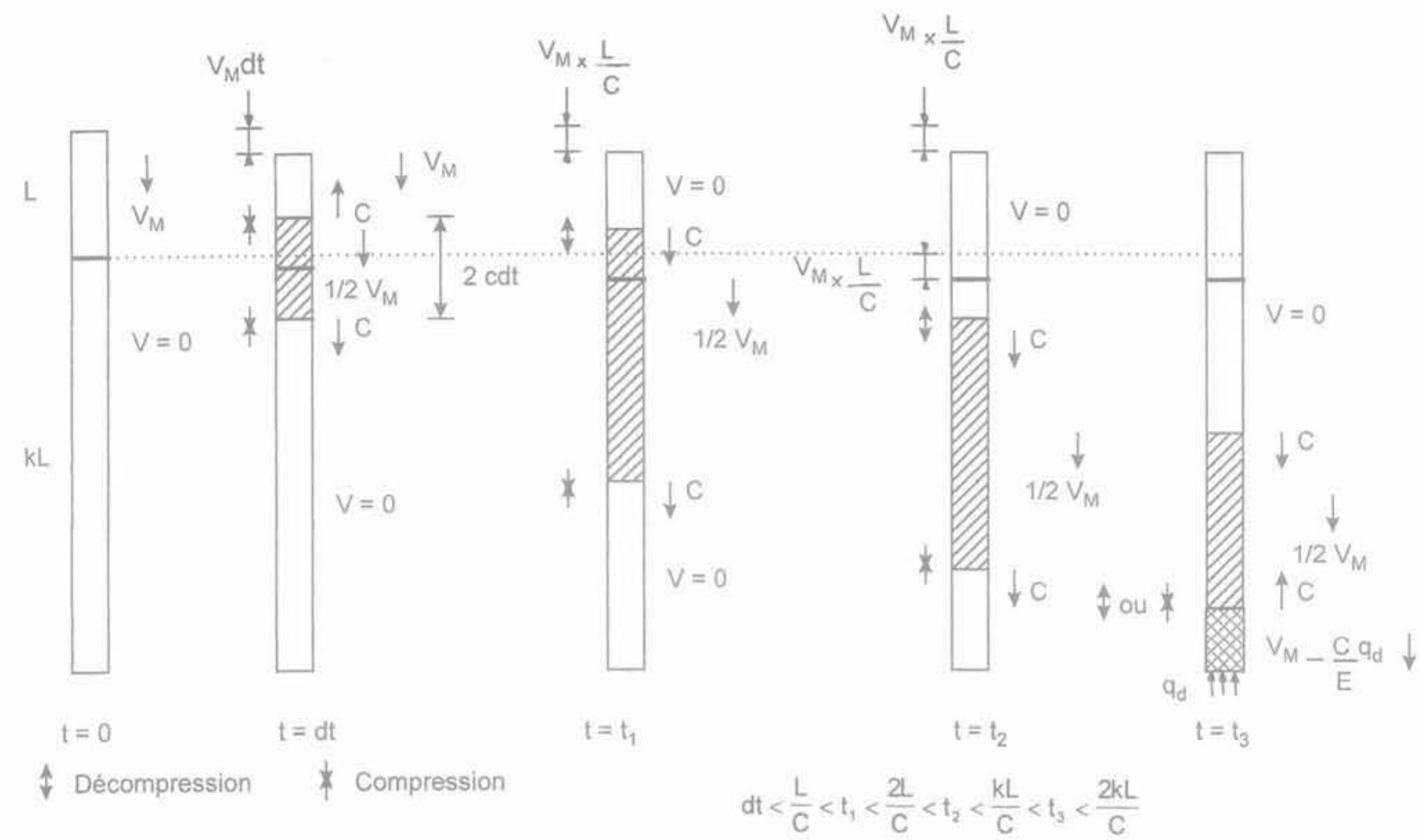

FiG.3 Choc d'un marteau et d'une tige de même section.

Impact of hammer and rod with the same section.

Ce raisonnement, déjà exposé, sous une forme un peu différente, dans de précédents articles (voir bibliographie), se fonde directement sur les principes de base établis par Newton et Hooke à la fin du XVII siècle.

Analysons maintenant l'utilisation de l'énergie en pointe pour la pénétration : nous voyons bien que l'on ne peut pas admettre, comme pour établir la formule des Hollandais, qu'une partie de l'énergie du mouton se volatilise au moment du choc, sans chercher à savoir où elle est passée!

L'onde de compression (onde rectangulaire «créneau») véhicule une énergie totale $E i=2 \frac{a L}{E} \sigma_{i}^{2}\left(\sigma_{i}=\frac{E V_{M}}{2 C}\right)$

Lorsqu'elle arrive en pointe, l'application des principes de la mécanique nous conduit aux résultats suivants (voir figure 4):

1) si $q_{\alpha}>2 \sigma$, aucun échange énergétique, donc aucune pénétration n'est possible ; l'onde incidente est réfléchie dans la tige en sens inverse en onde de compression de même intensité $\sigma_{i}$ (donc de même énergie $\left.2 \frac{a L}{E} \sigma_{i}^{2}\right)$;

2) si $\sigma_{i}<q_{d}<2 \sigma_{i}$ l'onde incidente est réfléchie en sens inverse en onde de compression d'intensité $\sigma_{r}=q_{d}-\sigma_{i}$; la pénétration a lieu à vitesse constante égale à $\frac{c}{E}\left(2 \sigma_{i}-q_{d}\right)$ pendant un temps égal à $2 \mathrm{~L} / \mathrm{c}$. Le même résultat est obtenu en écrivant que l'énergie de l'onde incidente $E_{i}=2 \frac{a L}{E} \sigma_{i}^{2}$ diminuée de l'énergie de l'onde réfléchie $E_{r}=2 \frac{a L}{E} \sigma_{r}^{2}$ est égale à l'énergie utilisée pour la pénétration (énergie (utile $n) \mathrm{Eu}=\mathrm{aq}_{\mathrm{d}} \mathrm{S}$ :

$$
2 \frac{a L}{E}\left(\sigma_{i}^{2}-\sigma_{r}^{2}\right)=2 q_{d} s \mathrm{~s}=\text { pénétration. }
$$

Dans ce cas; on a $0<\sigma<q / 2$, ce qui entraine que l'onde de compression réfléchie se propage (vers le haut) dans le mouton, auquel elle confère en se réfléchissant à sa partie supérieure une vitesse dans le même sens (vers le haut) égale en valeur absolue à $\frac{2 c}{E} \sigma_{r}$ : il y a rebond. Lorsque le mouton retombe sur la tige, le nouveau choc engendre une nouvelle onde de compression, de même énergie et de même intensité, mais vers le bas. Comme $\sigma_{r}<\frac{q_{d}}{2}$, cette nouvelle onde ne permet aucune pénétration: elle est à nouveau réfléchie. L'énergie de cette onde est donc emprisonnée dans la tige et s'épuise en d'inutiles allers et retours !



FIG.4 Onde rectangulaire de compression à la pointe. Rectangular stress-wave on tip. 
3) si $0<q_{d}<2 \sigma_{r}, \sigma_{r}$ est négatif et l'onde réfléchie est une onde de décompression. Elle ne peut se propager dans le mouton, qui n'est pas ( collé ») à la tige. Elle se réfléchit donc en onde de compression d'intensité $-\sigma_{r}$. Une nouvelle pénétration sera possible en pointe si $-2 \sigma_{r}>q_{d}$.

Posons, pour raisonner :

$$
\sigma_{i}=(n+1) q_{d} \quad \sigma_{r}=q_{d}-\sigma_{f}=-n q_{d}
$$

Pour toute valeur entière de $n$, la totalité de l'énergie de l'onde incidente initiale sera utilisée pour la pénétration, n mesurant le nombre d'arrivées d'ondes ayant chacune procuré une pénétration; en effet :

$$
\begin{gathered}
a q_{d} s=2 \frac{a L}{E} \sum_{n=0}^{n=n}\left(\sigma_{i}^{2}-\sigma_{s}^{2}\right)=2 \frac{a L}{E} q_{d}^{2} \sum_{n=0}^{n=n}\left[(n+1)^{2}-n^{2}\right]= \\
2 \frac{a L}{E} q_{d}^{2}(n+1)^{2}=E i
\end{gathered}
$$

Pour $\frac{\sigma_{f}}{q_{d}}$ non entier, égal à $\mathrm{n}+\alpha 1<\alpha<0$, on aura :

$$
a q_{\alpha} s=2 \frac{a L}{E} q_{a}^{2}\left[(n+\alpha)^{2}-\alpha^{2}\right]=E_{1}-2 \frac{a L}{E} q_{d}^{2} \alpha^{2}
$$

L'énergie perdue ( $($ emprisonnée ) en allers et retours dans la tige) est celle d'une onde " créneau $》$ d'intensité $\alpha q_{\alpha}$, étant compris entre 0 et 1 . II y a discontinuité chaque fois que la valeur de $\sigma / q_{d j}$ passe par une valeur entière.

On peut résumer ces résultats sur un graphique (voir figure 5) représentant le pourcentage d'énergie incidente utilisée pour la pénétration, $y=\frac{E u}{E i}=1-\frac{E r}{E i}$. en fonction de la valeur de $x=\frac{q_{d}}{\sigma_{f}}=q_{d} \times \frac{2 c}{E V_{M}}$ :

- pour $x>2$,

$$
y=0
$$

- pour $2>x>1 / 2$

- pour $1 / 2>x>1 / 3$,

$y=1-(x-1)^{2}$

- pour $\frac{1}{n}>x>\frac{1}{n+1}$

$y=1-(2 x-1)^{2}$

$y=1-(n x-1)^{2}$

Pour la pénétration $\mathrm{s}$, on aura, puisque pour $\mathrm{y}=1$, $\mathrm{E}_{1}=\mathrm{aq}_{\mathrm{d}} \mathrm{s}=\mathrm{MgH}$ :

- pour $x>2$

- pour $2>x>1 / 2$

$$
\begin{aligned}
& \mathrm{s}=0 \\
& a q_{\sigma} s=M g H\left[1-(x-1)^{2}\right]
\end{aligned}
$$

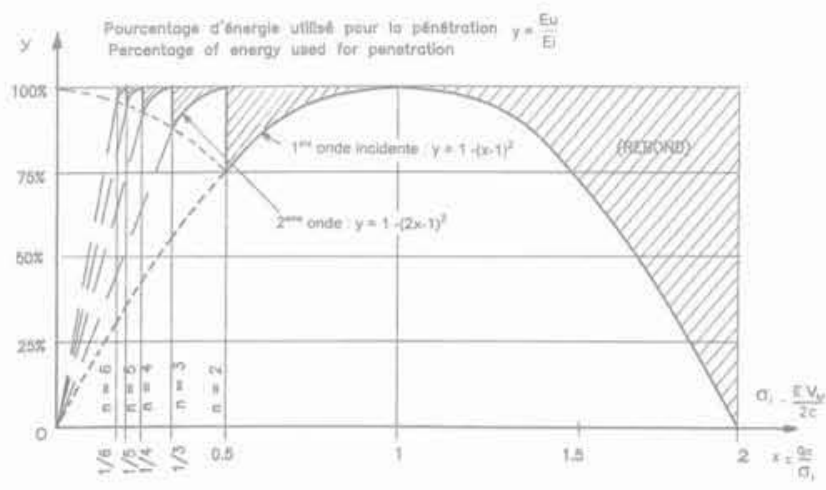

FG, 5 Pourcentage d'énergie utilisée pour la pénétration (onde rectangulaire).

Percentage of energy used for penetration (rectangular stress-wave)
- pour $\frac{1}{n}>x>\frac{1}{n+1} \quad \quad a q_{d} s=M g H\left[1-(n x-1)^{2}\right]$

Le pourcentage d'énergie incidente utilisée pour la pénétration varie de 0 à $100 \%$ suivant les valeurs du rapport $\frac{q_{d}}{\sigma_{i}}$; pour une valeur donnée de $q_{d}$, il dépend de $\sigma_{j}$, donc la vitesse $V_{M}$ du mouton à l'impact. Si $\underline{q_{d}}$

reste compris entre 0 et 1 , ce ( rendement $)$ reste compris entre 75 et $100 \%$, et il ne dépend pas de la longueur de la tige, ni par conséquent de sa masse totale.

On remarquera que lorsque le rapport $\frac{q_{d}}{\sigma_{i}}$ tend vers zéro, l'énergie tend à être entièrement utilisée pour la pénétration; mais le temps s'allonge indéfiniment, puisque le nombre n d'allers et retours de l'onde tend vers l'infini : la ( puissance » de l'énergie utilisée pour la pénétration tend vers zéro.

On remarquera aussi que pour ce cas limite (base de la tige "libre »), la pénétration de la tige (sa progression) se fait comme celle d'une chenille:

- arrivée de la $1^{\text {re }}$ onde $: \mathrm{s}_{1}=2 \mathrm{~L} \frac{V_{M}}{c}$

- arrivée de la $2^{\circ}$ onde : $\mathrm{S}_{2}=2 \mathrm{~L} \frac{V_{M}}{c}$ (1re onde réfléchie) - etc.

Les arrivées d'onde sont séparées par un temps $t=2 \frac{k L}{c}$.

La vitesse moyenne de progression de la tige est donc égale à $\frac{V_{M}}{k}, o u$, si $M$ est la masse du mouton et $\mathrm{m}$ celle de la tige, $V_{M} \times \frac{M}{m}$. Pour retrouver la valeur $V_{M} \times \frac{M}{M+m}$ de la formule des Hollandais, il faudrait supposer qu'au moment du contact, le mouton et la tige se soudent l'un à l'autre, de telle manière que le contact permette le passage des ondes quel que soit le signe de leur intensité.

\section{4}

\section{Pourquoi la formule des Hollandais est fausse}

Nous comprenons mieux, après cette analyse, pourquoi la " démonstration ») de la formule des Hollandais est fausse. Elle ne fait pas intervenir le temps : or le transfert d'énergie ne peut être abstrait du temps. Pendant le transfert, tout comme dans les instants qui le précèdent ou qui le suivent, l'énergie totale est conservée ; il y a bien conservation de la quantité de mouvement, mais aussi de l'énergie ; ne prendre en compte que la quantité de mouvement fait abstraction de l'énergie de compression, emmagasinée dans l'onde née du choc. Le raisonnement permettant de quantifier la perte d'énerqie:

$$
\left(1 / 2 M V_{M}^{2}-1 / 2 M V_{M}^{2} \times \frac{M}{M+m}=1 / 2 M V_{M}^{2} \times \frac{m}{M+m}\right)
$$

ne respecte pas les principes de la Mécanique rationnelle; il est arbitraire et ne peut conduire qu'à un résul- 
tat arbitraire. Cette critique fondamentale n'est guère présentée aux étudiants, à ma connaissance.

Une conséquence erronée qui, elle, n'échappe en génèral pas à l'examen critique de cette formule, vient du fait que le raisonnement étant indépendant du temps, il ne donne aucune limite pour la valeur de la force de pénétration de la tige : pour une pénétration nulle, la force devient infinie. C'est évidemment absurde au point de vue physique, et c'est pourquoi beaucoup de formules dérivées de la formule de base lui apportent des corrections, sans pour cela être plus valides au regard des principes de base de la mécanique.

\section{5}

\section{Analyse d'un cas plus général}

Le cas d'école discuté plus haut permet de raisonner simplement mais, s'il nous éclaire sur la réalité de l'enfoncement par battage d'une tige ou d'un pieu, il est assez éloigné des cas concrets, le mouton ayant toujours une section grande par rapport à celle de la tige. Le problème théorique du transfert d'énergie dans ce cas a été résolu en 1867 par Barre de St Venant, en supposant que le choc génère des ondes planes à la fois dans le mouton et la tige : il ne s'agit pas là, cette fois, d'une hypothèse arbitraire, mais d'une approximation acceptée. Nous allons étudier un autre cas qui se rapproche du cas théorique pour lequel la section du mouton est grande par rapport à celle de la tige. Considérons en effet une onde de choc de forme triangulaire, d'intensité maximale $\sigma_{\max }=\frac{E V_{M}}{C}=\rho c V_{M}$, de durée limitée à $3 \mathrm{~L} / 2 \mathrm{c}$, L étant la longueur de tige de mème masse que le mouton. L'énergie totale véhiculée par cette onde est égale à l'énergie du mouton à l'impact: $E_{\max }=\frac{a c}{E} \int_{0}^{\frac{3 L}{2 \epsilon}} \sigma^{2} d t=\frac{1}{2} \frac{L a}{E} \sigma_{\max }^{2}=\frac{1}{2} L a \rho V_{M}^{2}=\frac{1}{2} M V_{M}^{2}$

On peut voir sur la figure 6 que la courbe de contrainte théorique diffère assez peu de cette onde triangulaire pour $0<t<\frac{L}{C}$.

Posons $x=\frac{q_{d}}{\sigma_{\operatorname{mmx}}}$, et calculons le pourcentage d'éner-

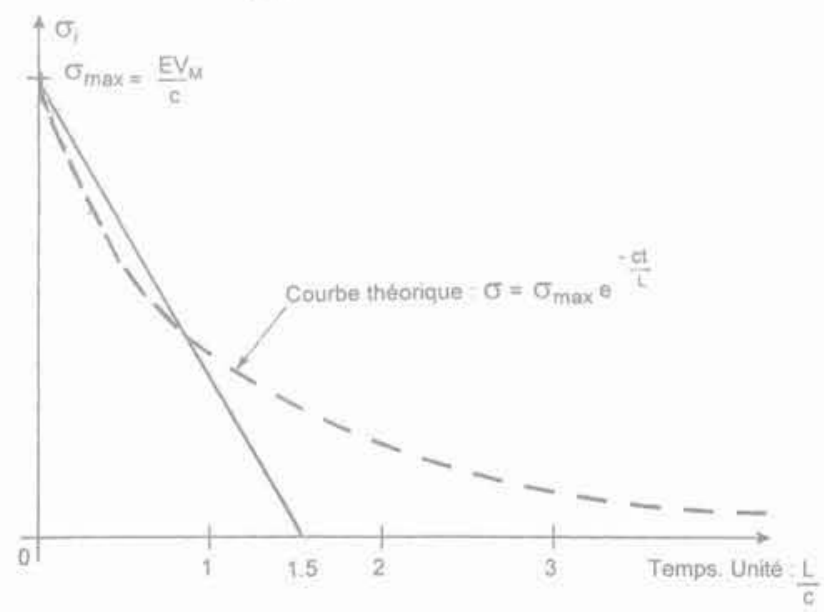

FiG.6 Onde triangulaire comparée à l'onde théorique.

Triangular stress-wave compared to the theoritical one. gie utilisée pour la pénétration, $y=\frac{E u}{E j}$, Ei étant l'énergie incidente, égale à $E_{\max }$

Pour la première onde incidente:

$$
E_{u}=\frac{a c}{E} \int_{0}^{\frac{3 L}{2 c}}\left(\sigma_{i}^{2}-\sigma_{r}^{2}\right) d t
$$

si $\sigma_{i}<q_{d} / 2, \sigma_{i}=\sigma_{r}$, donc $E u=0$, aucune pénétration n'est possible :

si $\sigma_{i}>q_{d} / 2: \sigma_{i}+\sigma_{f}=q_{d}$ et $\sigma_{i}-\sigma_{p}=2 \sigma_{i}-q_{d}$

or, pour $\sigma_{i}=q_{d} / 2, t=t_{0}=\frac{3 L}{2 c}\left[1-\frac{q_{d}}{2 \sigma_{\max }}\right]=\frac{3 L}{2 c}\left[1-\frac{x}{2}\right]$

Donc: $E u=\frac{a c}{E} \int_{0}^{t_{0}}\left(\sigma,-\sigma_{r}^{2}\right) d t=\frac{a c q_{d}}{E} \int_{0}^{t_{0}}\left(2 \sigma_{i}-q_{d}\right) d t$

avec $\sigma_{l}=\sigma_{\text {mix }}\left[1-t \times \frac{2 c}{3 L}\right]$.

Tous calculs faits : $\mathrm{Eu}=\frac{3}{2} \frac{a L q_{d}^{2}}{E} \times \frac{1}{x}\left[1-\frac{x}{2}\right]^{2}$

$$
\mathrm{Ei}=\frac{1}{2} \frac{\mathrm{aL}}{\mathrm{E}} \sigma_{\max }^{2}
$$

D'où : $y=\frac{E u}{E i}=3 x\left[1-\frac{x}{2}\right]^{2}$

Tant que la valeur maximale de la contrainte réfléchie reste, en valeur absolue, inférieure à $\frac{q_{d}}{2}$, seule la première onde incidente donne une pénétration (voir figure 7).

Pour $x>2$ : l'onde incidente est totalement réfléchie; aucune pénétration n'est possible:

pour $2>x>\frac{2}{3}$ : seule la première onde incidente donne une pénétration :

pour $\frac{2}{3}>x>\frac{2}{5}$ : l'onde incidente et la première onde réfléchie donnent une pénétration :

pour $\frac{2}{2 n-1}>x>\frac{2}{2 n+1}$ : $n$ ondes sont utilisées pour la pénétration.



F6. 7 Onde triangulaire - Ondes réfléchies $\left(q_{d} / \sigma_{\max }=2 / 7, n=3\right)$.

Triangular stress-wave - Reflected stress-wave. 
On trouve que la fonction y $(\mathrm{x})$ est la suivante :

$$
\begin{array}{cc}
x>2 & y=0 \\
2>x>\frac{2}{3} & y=3 x\left(1-\frac{x}{2}\right)^{2}
\end{array}
$$

(n entier positif) $x=\frac{2}{2 n+1}$

$$
\begin{gathered}
y=\frac{24}{(2 n+1)^{3}} \sum_{n=1}^{n=n} n^{2}=\frac{4 n(n+1)}{(2 n+1)^{2}}=1-\frac{x^{2}}{4} \\
\frac{2}{2 n-1}>x>\frac{2}{2 n+1} \\
y=3 x\left[\left(1-\frac{x}{2}\right)^{2}+\left(1-\frac{3 x}{2}\right)^{2}+\ldots+\left(1-\frac{2 n-1}{2} x\right)^{2}\right]
\end{gathered}
$$

La figure 8 récapitule les résultats du calcul. Sur le même diagramme, on a fait figurer la courbe correspondant à la première onde incidente pour une onde théorique non simplifiée, le rendement y étant alors donné par $y=4 x\left[\left(1+\frac{x}{2}\left(\log \frac{x}{2}-1\right)\right]\right.$ (Gonin, 1978).

La différence entre les deux courbes représente au. maximum un écart de $15 \%$; le a rendement $»$ maximal pour la première onde incidente étant de $88,9 \%$ pour l'onde triangulaire et de $81,5 \%$ pour l'onde théorique.

Pour une onde triangulaire, si $0<x<\frac{2}{3}$, on a, avec une bonne approximation : $y=1-\frac{x^{2}}{4}$.

En terme de pénétration totale $S$, on pourra donc écrire :

$$
\begin{array}{ll}
\text { si } 2>x>\frac{2}{3} & a q_{d} s=E i \times 3 x\left(1-\frac{x}{2}\right)^{2} \\
\text { si } \frac{2}{3}>x>0 & a q_{d} s=E i\left(1-\frac{x^{2}}{4}\right) \\
\text { avec } x=q_{d} \frac{c}{E V_{M}} & \text { et } \quad E i=\frac{1}{2} \frac{a L}{E} \sigma_{m}^{2}=\frac{1}{2} M V_{M}^{2}
\end{array}
$$

Il est possible, sans grande difficulté, de traiter de la méme façon le cas de la courbe de contrainte théorique du choc d'un mouton de grande section par rap-

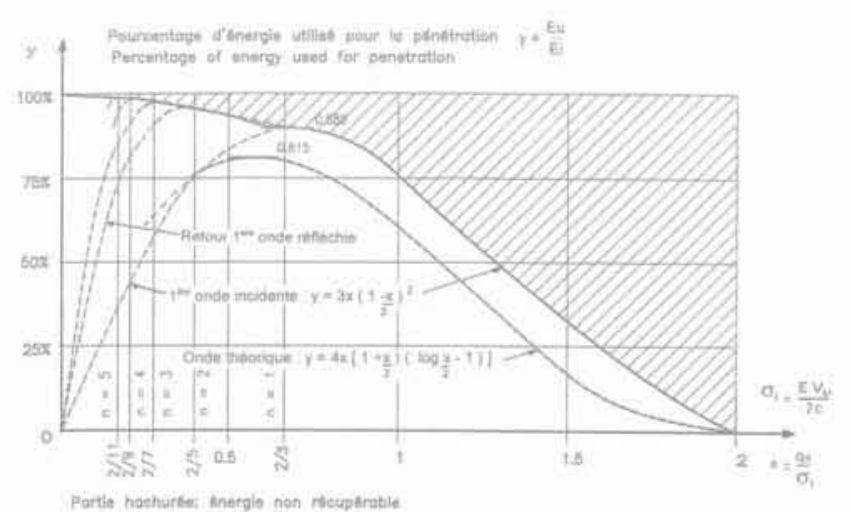

FG. B Pourcentage d'énergie utilisée pour la pénétration (onde triangulaire).

Percentage of energy used for penetration (triangular stress-wave). port à celle de la tige. On peut aussi, en utilisant les résultats ci-dessus, linéariser la courbe de contrainte de l'onde incidente en considérant une succession d'ondes triangulaires.

\section{6}

\section{Amortissement : ébauche d'une approche raisonnée}

Nous avons vu que l'erreur commise dans l'établissement de la formule des Hollandais tenait à la manière arbitraire de considérer une perte d'énergie du système mouton-tige. Toutefois, les corps n'étant pas parfaitement élastiques, il peut paraitre légitime de prendre en compte un amortissement des ondes, surtout lorsque leurs trajets s'allongent du fait des réflexions, indépendamment, bien entendu, de l'action du sol encaissant le long de la tige, que nous ne considérons pas dans cette analyse.

Il est habituel, et conforme aux données de l'observation, de faire alors intervenir un amortissement de type visqueux, c'est-à-dire proportionnel à l'intensité de l'onde ou à la vitesse particulaire :

$$
\frac{d v_{p}}{v_{p}}=-\alpha c d t
$$

$\alpha$ : coefficient d'amortissement ; cdt : trajet de l'onde pendant dt.

Cela revient à considérer que l'onde de contrainte est affectée, dans son trajet, d'un coefficient minorateur dépendant du temps, donc du parcours de l'onde:

$v_{p}=v_{p o}\left[e^{-\alpha(t-t o)}\right]$

$$
\text { où : } \sigma=\sigma_{0}\left(e^{-\alpha}\right) \quad \mathrm{I}=\mathrm{c}(\mathrm{t}-\mathrm{to})
$$

1: longueur parcourue par l'onde pendant le temps $\mathrm{t}-\mathrm{to}$.

Nous avons montré plus haut qu'il suffit, en règle générale, de trois à quatre allers et retours de l'onde incidente pour que la plus grande part d'énergie utilisable ait servi à la pénétration; tout est joué en un à deux centièmes de seconde. II y a tout lieu de penser. qu'en dehors de l'action du sol le long de la tige, fort peu d'énergie est perdue par l'amortissement de l'onde dans son trajet; la plus grande part l'étant dans les ondes $\alpha$ emprisonnées $x$, trop peu énergétiques pour la pénétration, et condamnées par conséquent à se transformer en chaleur au cours d'infinis allers et retours. C'est aussi le cas dans le choc de deux boules de billard, dont l'énergie cinétique totale n'est pas la même après leur rencontre qu'avant : pour établir la formule des Hollandais, on s'est peut-ètre inspiré, bien mal à propos, de ce cas, dont l'étude théorique est complexe (le théorème de Clapeyron, par exemple, ne s'applique pas).

\section{Conclusion}

En conclusion, je m'adresserai, d'une part, à ceux qui ont la lourde tâche, au sein de nos illustres Universités ou de nos irremplaçables grandes Écoles, de former les futurs ingénieurs aux techniques appliquées ; d'autre part, à leurs brillants élèves. 
Aux premiers, une objurgation : de grâce, que la formule des Hollandais ne soit plus enseignée que de manière critique, à titre de contre-exemple ; aux seconds, un conseil : ne prenez pas toujours pour argent comptant ce qui est écrit dans les livres, ou même ce qui sort de la bouche de vos éminents professeurs : il y a beaucoup de perles, mais parfois quelques crapauds !

\section{Bibliographie}

Gonin H. - « Etude theorique du battage des corps élastiques élancés. D Annales de I'ITBTP; série Sols et Fondations, $n^{\circ} 155,1978$.

Gonin H. - « Réflexions sur le battage des pieux. is Revue française de Géotech. nique, $n^{\circ} 9,1979$.
Gonin H. $-\alpha$ Du pénétromètre dvnamique au battage des pleux. $\rightarrow$ Revue française de Géotechnique, n 76, 1996.

Newton I. - Philosophiae naturalis principia mathematica, Londres, 1687. D'après Encyclopaedia Universalis, Tome 16, Éd. 1990.
De Saint-Venant - « Mémoire sur le choc longitudinal de deux barres élastiques. x Journal de mathématiques, $2^{\text {E }}$ série XII 1867, p. 237-276. 